\title{
Analysis and synthesis of regime controllability of heat supply systems
}

\author{
Zoya Shalaginova, ${ }^{1, *}$ \\ ${ }^{1}$ Melentiev Energy Systems Institute of the Siberian Branch of the Russian Academy of Sciences, \\ 664033, Lermontova str., 130, Irkutsk, Russia
}

\begin{abstract}
A mathematical model of the heat and hydraulic regimes (HHR) of the heat supply system (HPS) is given in time, taking into account the transport delay, disturbing, and control actions. Based on the simulation of the HHR, the indicators of consumer security integrated over the billing period were developed. The analysis of heat deficit indicators allows us to adopt a decision on the placement of additional control units to improve the regime controllability. The approach to the task of placing intermediate stages of regulation based on the construction of path matrices from final consumers in the direction of the reverse flow of the coolant is given. The model is implemented in the information-computational complex (ICC) "ANGARA-TS" for the calculation of the HHR of HPS, which is developed in the Melentiev Energy Systems of SB RAS.
\end{abstract}

\section{Introduction}

Modern heat supply systems (HPS) have been developing as spatially distributed complex systems of centralized supply, being characterized by the following structural and regime aspects: the joint operation of heat sources (HS) for common heat networks; the presence of intermediate control stages - central heating points (CHP), which allow to implement the functioning modes in networks of different levels (main, distribution), which are very different both in terms of organization (quantitative, qualitative regulation) and the parameters of the coolant. The study of the regime controllability of such systems under various operating conditions is a necessary element for assessing the feasibility of decisions taken at the design and operational levels, in the reconstruction and planning of regimes.

According to the existing terminology, regime controllability is the property of the system to maintain the required mode by means of control. The essence of management is a purposeful change in the state of the HPS to provide all consumers at each time with the required amount of heat of a given quality (the need for which can vary in accordance with changes in the weather conditions, the regime of hot water consumption, etc.). At the same time, the following should be observed: technical requirements for maintaining all the parameters of the coolant (pressures, costs, and temperatures) at the anchor points of the HPS within the specified limits; conditions of safety and trouble-free operation; economic criteria for minimizing the costs of production, transport and distribution of heat.

\footnotetext{
* Corresponding author: shalaginova@ isem.irk.ru
} 
The problem of controllability in HPS is associated with the rapid growth of their scales and complexity with insufficient level of design, operation 6 and technical equipment, especially transport and distribution systems [1]. Due to the historical development of the HPS, their structure, as a rule, did not have a clear hierarchy of construction, and therefore existing HPSs only have partial controllability under normal conditions and become uncontrollable if they are violated.

Requirements for the structure of HPS. The principles of building HPS in Russia were already researched in the $30 \mathrm{~s}$ of last century. The first proposals for the rational construction of heat networks were made during the development of the Moscow heat distribution scheme, when a ring scheme of heat networks was proposed [2]. According to the current Russian concept of constructing automated heat supply systems taking into account reliability and controllability [3], large HPS with several HS and extensive looped heat networks, with a large number of connected heterogeneous consumers, should have several levels of management for the coordinated work of all HPS links: in HS; in the intermediate nodes of the heat network (CHP); in individual heat points (IHP) and in subscriber installations. When organizing a multistage control, each stage facilitates the work of the following.

Recently, there has been much talk about the imperfection of the HPS structure being adopted in Russia, with the presence of a central heating station [3], due to large losses of heat and water in distribution networks and low efficiency of the equipment installed [4-6]. The pre-property of heat supply systems with IHPs is the finer adjustment of the thermal regime of local heat consumption systems. These advantages should most fully manifest themselves in the new construction, provided that the equipment will be fully installed, debugged, put into operation, and properly operated. It must also be remembered that no country in the world can match Russia in terms of centralized heat supply. The consumption of heat energy only in Moscow exceeds its total consumption in Holland and Sweden together, and in St. Petersburg - higher than in Finland or Denmark [7]. The large scale and complexity of Russian HPSs determine the important role of CHP presence in them, especially with large differences in geodetic marks. Of course, energy-saving HPS should work with IHP. However, this does not mean that the CHP should be closed. They serve as a hydraulic stabilizer and simultaneously separate the HPS into separate subsystems. At the same time, a two-pipe gasket can be retained behind the CHP, but the distribution heat networks (DHN) can operate at reduced parameters in comparison with the main. This makes it possible to reduce heat losses in the secondary circuit because of leaks and ensure the stable operation of the IHP, excluding their rigid mutual influence along the hydraulic path.

\section{Problem statement}

Strengthening of material, economic, and technological ties, as HPS progressed, the application of a systematic approach to designing and managing their functioning became extremely topical, since the decisions applied to each of the links separately are not optimal for the system as a whole. Often such solutions do not meet the requirements of reliability and manageability, and sometimes are unrealizable, especially in developing HPS with the existing structure. The annual increase in the connected load requires an informed decision on the issues of their reconstruction and expansion.

At present, taking into account the constantly growing prices for thermal energy, activities on introduction of accounting and autonomous regulation tools are being activated. In turn, the problem of economic responsibility for excess heat losses in networks, which can reach $30 \%$ of the released amount of heat, is aggravated. There are more stringent requirements for the HPS management, including the ability to quickly 
assess the dynamics of changes in loads during the day, weeks, seasons of the year, the ability to calculate and assign appropriate modes of operation of the system as a whole. These problems are especially aggravated in the new economic relations and require new approaches and methods for their solution with maximum consideration for the factors listed above.

Indicators for assessing the quality of functioning for the analysis of HPS controllability. At present there are no generally accepted quantitative indicators of the regime controllability of the HPS. At the same time, it is possible to assess the controllability of the HPS in terms of the quality of the functioning in various modes. The quality of the HPS functioning is assessed by the following criteria: the efficiency of the regimes and the degree of deviation of the coolant parameters delivered to consumers from the required values $[8,9]$.

For most consumers, it does not matter which combination of parameters (flow and temperature) will provide the required load. The ultimate goal is to ensure that the air temperatures in the premises and water in the heat water supply (HWS) are within the specified limits. In this regard, instead of the criterion of the degree of deviation of the parameters, one can consider the degree of providing consumers with the required amount of heat. It would be wrong to use the average for the settlement period for this purpose. It may turn out to be close to what is required, although at some points the consumer might receive enough heat, while others might get it in excess. Therefore, we should use indicators of the degree of security at each point in time of the calculation period with the subsequent calculation of integral indicators. This circumstance requires the involvement of dynamic models.

A mathematical model and a technique for calculating dynamic thermohydraulic regimes of arbitrary configuration and structure with various schemes of attaching heterogeneous loads (heating, ventilation, hot water) $[13,14]$ to the foundations of the theory of hydraulic circuits have been developed at the MESI of SB RAS [10-12]. The model allows to calculate the distribution of hydraulic parameters (costs and pressures) and the "temperature field" for all elements of the design scheme. The calculation takes into account cooling by length, transport delay with various perturbations in HS, CHP, pumping stations (PS), consumers (IHP), and heat network sections [15, 16]. To model the operation of large HPSs with intermediate control nodes, the principle of multilevel modeling is used [17-19]. For the modeling of CHP and IHP, a mathematical model and a technique for calculating thermal-hydraulic modes of thermal points have been developed [16, 20].

Simulation of HPS functioning modes during the settlement period (for example, the heating season) is carried out by setting two types of scenarios:

- disturbances: changes in certain times of the temperature of outdoor air, water withdrawal by hot water supply, network topology (in emergency situations);

- controls: changes at certain times of the temperature, pressure, and flow rate of the coolant at the exit from the HS and CHP, the pressure of the PS, the resistances of the sections with regulators, the network topology (with targeted switching) in accordance with the disturbing effects.

The HPS reacts to disturbing and control actions by changing parameters of the regimepressures, flow rates, and temperatures at the sections and at the nodes of the system. The composition and the set of these changes depend both on the set and magnitude of the perturbations, and on the properties of the HPS itself, its structure, the presence of a central heating station and IHP, the characteristics of regulators, and design parameters of the network. To assess the controllability of a HPS, it is necessary to know to what extent the parameters of the regimes depend on external disturbances and control actions, determine the response of HPS's individual elements and the time of its onset to various kinds of effects. It is known that the pressure in the network propagates approximately at the speed 
of sound, so the reaction to changes in parameters affecting the hydraulic regime (pressure, flow, resistance of the sections with regulators) will occur almost instantaneously and simultaneously in all HPS nodes. At the same time, the temperature transfer is carried out together with the mass of the coolant, i.e. with the speed of its movement, and the response to the temperature changes will occur with a delay equal to the time of transport of the coolant from the disturbance node to the node under consideration. In the end, any changes in the parameters of the HPS affect the quality of heat supply to consumers.

The quality of heat supply to a particular $(i$-th) consumer can be estimated by the degree of deviation of the amount of heat received by it $\theta_{i}$ from the required one $\theta_{i}^{*}$. The degree of security of the $i$-th consumer at the moment of time $\tau$ can be expressed by the following characteristic:

$$
\Omega_{i}^{\text {म }}(\tau)=\left(\theta_{i}^{*}(\tau)-\theta_{i}(\tau)\right) \cdot \vartheta, \quad i \in I_{2},
$$

where $I$ is the set of sections of the circuit $I=I_{1} \cup I_{2} \cup I_{3} ; I_{1}, I_{2}, I_{3}$ are the subsets of sections of the network, consumers, and sources; $\vartheta$ is the weight coefficient; $\vartheta=1$ if those $\left(\theta_{i}^{*}(\tau)-\theta_{i}(\tau)\right)>0$. the consumer feels a shortage of heat; $\vartheta=0$ if $\left(\theta_{i}^{*}(\tau)-\theta_{i}(\tau)\right) \leq 0$, i.e. the consumer is provided with thermal energy in the right amount or receives it in excess.

The total heat deficit of the $i$-th consumer for the calculation period $T$ under the $k$-th control scenario can be determined by the following relationship:

$$
\Psi_{i}^{\mathrm{\jmath}(k)}=\int_{0}^{T} \Omega_{i}^{\mathrm{\jmath}(k)}(\tau) d \tau, \quad i \in I_{2}
$$

Total heat deficit for all consumers under the $k$-th scenario:

$$
D^{(k)}=\sum_{i \in I_{2}} \Psi_{i}^{\mathrm{д}(k)},
$$

Average deficit for the calculation period $T$ under the $k$-th scenario:

$$
\Psi_{i_{\mathrm{cp}}}^{\mathrm{\nexists}(k)}=\frac{1}{T} \int_{0}^{T} \Omega_{i}^{\mathrm{z}(k)}(\tau) d \tau, \quad i \in I_{2}
$$

Repeating the calculations $N$ times for different scenarios of external disturbances with the given control rules, it is possible to calculate the average expected deficit of the $i$-th consumer:

$$
M\left[\Psi_{i_{\mathrm{cp}}}^{\text {д }}\right]=\frac{1}{N} \sum_{k=1}^{N} \Psi_{i_{\mathrm{cp}}}^{\text {д(k) }}, \quad i \in I_{2}
$$

Dispersion:

$$
D\left[\Psi_{i_{\mathrm{cp}}}^{\text {д }}\right] \approx \frac{1}{N-1} \sum_{k=1}^{N}\left[\left(\Psi_{i_{\mathrm{cp}}}^{\mathrm{д}(k)}\right)-\left(\frac{1}{N} \sum_{k=1}^{N}\left(\Psi_{i_{\mathrm{cp}}}^{\text {д(k)}}\right)\right)\right]^{2}, \quad i \in I_{2}
$$

The integrated indicators of excess heat energy released to consumers are similarly calculated for the calculated period of time.

Synthesis of the HPS regime controllability. After carrying out simulation calculations of the system operation for the elapsed period and obtaining the values of the indicators (3) 
- (6), we can proceed to the issue of placing additional control nodes. The normal mode of operation of the HPS is defined as the design mode of operation under normal conditions, when the values of the specified operating parameters are ensured within the specified limits. The system is manageable if it is capable of realizing all design modes. Synthesis of regime controllability consists in choosing solutions for the development of the system that will ensure the implementation of the most beneficial modes and facilitate their change in time.

The purpose of the options for increasing the degree of controllability of the HPS being studied can be accomplished using the following approach.

Preliminary, we set the control scenario for the past heating season and carry out calculations of the thermal-hydraulic regimes according to the model [13]. Calculating the values of deficits for all users of the system using equations (1)-(6), we rank the consumers, within each DHN, from the point of view of their provision with the required amount of heat for the billing period.

Further we will work with each DHN separately. All the nodes of the branching of the network diagram can be considered as generalized consumers that precede the flow to certain groups of real consumers. Using the property of hierarchical subordination of consumers in this sense, it is possible to outline the locations of additional control nodes (CHP) to minimize the deficit in real consumers of the lower levels. The algorithm consists in constructing paths from consumers in the direction opposite to the flow of the heat carrier obtained from the flux distribution calculation (Fig. 1).

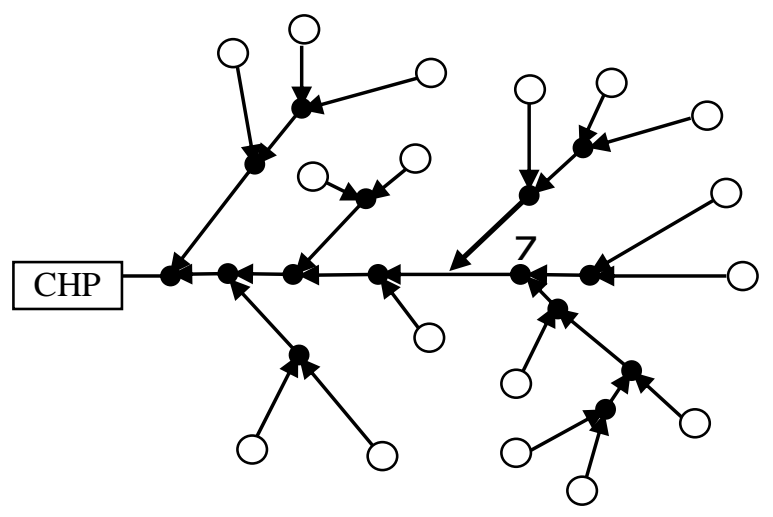

Fig.1. Algorithm for placing additional control nodes.

The value of the consumer deficit is assigned to the supply site. In the communication node of the sections belonging to the paths from different consumers (let's call its node $z$ ), the heat provision for all branches is summed up and the sums of deficiencies $\sum_{1}^{L} D_{z}^{(k)}$, calculated by the formula (2) are summed, where $L$ is the number of branches from the node $Z$. Then the average total deficit of all consumers associated with the node $(z)$ for the billing period:

$$
D_{z}^{(k)}{ }_{\mathrm{cp}}=\frac{1}{T}\left(\sum_{1}^{L} D_{z}^{(k)}\right)
$$

For nodes connecting several dysfunctional branches (three or more), the required thermal load at the node and the average expected deficit in different $M\left[D_{z}^{(k)}\right.$ cp $]$ scenarios are calculated, according to formula (5). The installation of the central heating station is 
planned if $M\left[D_{z}^{(k)}{ }_{\text {cp }}\right]$ it is more than $10 \%$ of the required load, and this load lies in the range from 2 to $10 \mathrm{MW}$, which is the optimum load of the central heating station.

Thus, several different automation options are planned, which are then checked to ensure the regime controllability of indicators (3)-(5) and are compared by cost and payback.

\section{Conclusion}

1. Improving the manageability of modern HPS require a set of activities related to both the introduction of technical means for measuring, regulating, and recording heat supply and consumption, and developing methods and mathematical models for the analysis and synthesis of regime controllability.

2. For the quantitative assessment of regime controllability, integral indicators of consumer security for the settlement period are proposed, based on the model of thermalhydraulic HPS regimes in time.

3. A new approach is proposed for solving the problem of the synthesis of regime controllability of large heat supply systems, based on the use of developed indicators of consumer security.

The research has been conducted within the framework of the scientific project III.17.4.3., Melentiev Energy Systems Institute of SB RAS, Reg. No. AAAA-A17117030310437-4.

\section{References}

1. Z. I. Shalaginova, Methodological issues of reliability research for large energy systems, 49, 504 (1997)

2. N. K. Gromov, Urban Heating Systems (Energiya, Moscow, 1974)

3. S. A. Chistovich, V. K. Averyanov, Yu. Ya. Tempel, S. I. Bykov, Automated systems of heat supply and heating (Leningrad, Stroyizdat, 1987)

4. A. N. Davydov, Heat Supply News, 12, 52 (2004)

5. M. I. Dubson, Heat supply news, 4, 92 (2008)

6. V. I. Livchak, Energy Saving, 1, 36 (2008)

7. I. A. Bashmakov, Energy Saving, 2, 46 (2010)

8. N. I. Voropai, N. N. Novitsky et al., Methods of controlling physical and technical systems of power engineering under new conditions (Nauka, Novosibirsk, 1995)

9. Z. I. Shalaginova, Thermal Engineering, 59(5), 408 (2012)

10. A. P. Merenkov, V. Ya. Khaselev, Theory of hydraulic circuits (Nauka, Moscow, 1985)

11. N. N. Novitsky, M. G. Sukharev, A. D. Tevyashev and others, Pipeline energy systems. Methodical and applied problems of mathematical modeling (Nauka, Novosibirsk, 2015)

12. N. N. Novitsky, V. K. Averyanov, A. V. Alekseev, et al., Pipeline Energy Systems: Development of Methods of Mathematical Modeling and Optimization (Nauka, Novosibirsk, 2008)

13. Z. I. Shalaginova, Thermal Engineering, 56(12), 1016 (2009) 
14. A. V. Alekseev, O. A. Grebneva, N. N. Novitsky and others, Research and development of the SB RAS in the field of energy-efficient technologies (2009)

15. V. V. Tokarev, Z. I. Shalaginova, Thermal Engineering, 63(1), 68 (2016)

16. Z. I. Shalaginova, Thermal Engineering, 63(3), 222 (2016)

17. N. N. Novitsky, V. V. Tokarev et al., Proceedings of the Russian Academy of Sciences. Power Engineering, 1, 12 (2018)

18. N. N. Novitsky, A. V. Alekseev, O. A. Grebneva, Energy (in press), (https://doi.org/10.1016/j.energy.2018.02.070, 2018)

19. V. V. Tokarev, Z. I. Shalaginova, Mathematical models and methods of analysis and optimal synthesis of developing pipeline and hydraulic systems, X12, 300 (2010)

20. A. A. Atavin, N. N. Novitsky, M. G. Sukharev, Pipeline energy systems: mathematical and computer technologies of intellectualization (Nauka, Novosibirsk, 2017) 